\title{
Les investissements publics chinois dans les filières agricoles ivoiriennes
}

\author{
Xavier Aurégan* \\ Centre de recherches et d'analyses géopolitiques (CRAG), université Paris 8, 2 rue de la Liberté, 93526 Saint-Denis, France
}

\begin{abstract}
Résumé - Cet article propose une analyse des différents investissements publics chinois dans l'agriculture ivoirienne. Ces investissements directs à l'étranger, non associés à de l'aide au développement, recoupent des acteurs aux stratégies parfois antagoniques. Sont-elles indépendantes de l'État chinois ? Si ce type d'investissement reste secondaire dans les relations entre Pékin et Abidjan et entre la Chine et l'Afrique, il monte toutefois en puissance. En Côte d'Ivoire, ces investissements directs à l'étranger concernent exclusivement les filières cacao et hévéa et impliquent deux géants de l'agroalimentaire et du négoce chinois. Pierre angulaire de la relation sino-africaine post-1994, ces acteurs publics se trouvent au cœur des stratégies commerciales de la Chine et de ses forums de coopération Chine-Afrique (FOCAC). À leur niveau, ils participent en définitive à la financiarisation du secteur agricole et à la montée en puissance de la dorénavant mondialisée « China Corp. ».
\end{abstract}

Mots clés : Chine / Afrique / Côte d'Ivoire / agriculture / investissement

\begin{abstract}
The Chinese government investments in Ivorian agricultural sectors. This paper analyses investments from the People's Republic of China in the Ivorian agriculture. Foreign direct investments, not linked to aid, involve various stakeholders and their conflicting strategies. Are they independent of the Chinese State or its provinces? This type of investment is secondary in the Beijing-Abidjan and China-Africa relationships; however, it is growing. In Ivory Coast, these investments are exclusively related to cocoa and rubber industries and involve only two Chinese giants of food industry and trading. Cornerstone of the post-1994 Sino-African relations, these actors are at the heart of Chinese business strategies and its cooperative Forum on China-Africa Cooperation (FOCAC). Ultimately, at their level, they contribute to the financialization of the agricultural sector and to the rise to power of the now globalized "China Corp.".
\end{abstract}

Keywords: China / Africa / Ivory Coast / agriculture / investment

\section{Introduction}

En Afrique subsaharienne, les différentes formes de présence chinoise sont abondamment commentées et engendrent une riche littérature (Chaponnière et Gabas, 2012). Toutefois, cette dernière est souvent limitée aux migrants et aux secteurs de prédilection des acteurs chinois : les infrastructures et les activités extractives. Hormis quelques chercheurs francophones, comme Gabas (2014), et anglophones, comme Brautigam (2015), les productions scientifiques concernant l'agriculture (Chaponnière, 2011) restent limitées, notamment aux phénomènes les plus visibles : les accaparements de terres. Il s'agit ici plutôt de s'intéresser aux investissements agricoles chinois en posant les questions suivantes : qui sont ces investisseurs chinois ? Ces investissements sont-ils le fait de l'État chinois lui-même, qui se sert éventuellement de ses entreprises à capitaux publics pour

\footnotetext{
$\bar{*}$ Auteur de correspondance : xavierauregan@hotmail.com
}

mener à sa place ses propres stratégies, ou bien des acteurs qui sont certes publics, mais indépendants des politiques gouvernementales ? Dans les deux cas et bien qu'aucune réponse ne soit apportée ici, une question additionnelle se posera à court terme : ces investissements servent-ils les intérêts locaux, africains, ceux des gouvernants et/ou des gouvernements endogènes ?

Avec $15 \%$ du produit intérieur brut (PIB) africain et 50 à $80 \%$ de la population active selon les pays, l'agriculture reste prépondérante dans les sociétés au Sud du Sahara, et ce malgré la tertiarisation des économies. Avec une économie extravertie, historiquement dépendante des cours des marchés et des rentes agricoles afférentes, la Côte d'Ivoire ne fait pas exception : les produits agricoles représentent $70 \%$ des recettes d'exportation. La Chine populaire, qui est présente depuis les années 1950 en Afrique - et depuis 1983 en Côte d'Ivoire (Aurégan, 2013) -, apporte une expérience différente de celle des partenaires traditionnels, dont la France. Cette expérience plurielle est historiquement diffusée par l'aide au développement et ses représentants (techniciens, ingénieurs et agronomes). Avec la 
diversification des acteurs chinois en Afrique, les modes opératoires et les finalités se complexifient dans ce paysage bigarré où s'entrecroisent ambassades, puissants groupes publics et migrants-investisseurs, ces derniers participant « par le bas » à la relation économique sino-africaine.

En Côte d'Ivoire, les séries de réformes chinoises des décennies 1980 et 1990 se matérialisent via l'une de ses principales formes d'intervention en Afrique : les investissements directs à l'étranger (IDE), encore appelés investissements directs internationaux (IDI) par l'Organisation de coopération et de développement économiques (OCDE, 2008). En plaçant l'agriculture comme priorité dans ses relations avec le continent africain, et notamment lors des Forums de coopération Chine-Afrique (FOCAC), Pékin entend contribuer au développement de ce secteur qui reste primordial dans les économies formelles et informelles des États subsahariens.

Après la présentation de la méthodologie, cet article présente les résultats obtenus en trois parties. La première présente une typologie des acteurs et des modes d'intervention chinois en Afrique. La deuxième discute l'importance de l'agriculture dans les relations bilatérales sino-ivoiriennes. La troisième analyse plus en détail deux filières agricoles présentant un intérêt particulier, le cacao et l'hévéa.

\section{Méthodologie}

Les résultats analysés ci-dessous proviennent d'une recherche encadrée par le Centre de coopération internationale en recherche agronomique pour le développement (CIRAD) et financée par le Technical Centre for Agricultural and Rural Co-operation ACP-EU (CTA). Après six années de recherches sur les relations sino-ivoiriennes (Aurégan, 2016b), une étude de terrain a été réalisée fin 2014 à Abidjan. Des dizaines d'entretiens ont été effectués avec les principaux acteurs concernés : le ministère de l'Agriculture ivoirien, le Bureau économique et commercial chinois, les investisseurs chinois, les représentants syndicaux et associatifs des filières concernées, les institutions de recherche et les centres techniques agronomiques et agricoles ivoiriens, les centres de recherche français, etc.

\section{Typologie des acteurs et des modalités d'intervention chinois}

Officialisées le 2 mars 1983, les relations sino-ivoiriennes tendent à se diversifier et à augmenter dans les années 2000. Cette décennie débute par le premier FOCAC de 2000 et va voir, année après année, une forte évolution des rapports entre la Chine et l'Afrique, Côte d'Ivoire comprise. Les impacts des réformes chinoises des années 1980 et 1990 se caractérisent par l'augmentation des échanges, des flux et des relations, qu'elles soient financières, économiques, culturelles, sociales ou diplomatiques. Ce rehaussement tous azimuts est permis par l'instauration d'instruments, d'institutions et de cadres normatifs spécifiquement orientés vers l'outre-mer, et notamment l'Afrique. Evoquer les investissements publics chinois dans les filières agricoles ivoiriennes suggère de pouvoir différencier, en amont, ce qui relève des interventions privées de ce qui relève de la coopération publique bilatérale. Les premières ne sont pas analysées ici et constituent les investissements réalisés à l'étranger par des ressortissants chinois dans des sociétés de droit ivoirien créées in situ. La seconde modalité, quant à elle, est au cœur de la politique africaine chinoise depuis le milieu des années 1990.

Pour la Chine, ses représentants et ses ressortissants, la palette de modalités d'intervention tend à s'enrichir depuis les réformes des années 1980. Parfois, ces modalités s'imbriquent partiellement et contribuent aux critiques portant sur la - faible - transparence de l'intervention chinoise en Afrique. C'est spécifiquement le cas lorsque investissements publics et coopération sont enchevêtrés. Ce que nous nommons « coopération » intègre l'aide au développement, une aide chinoise qui est liée au sens de l'OCDE (2008b), et qui, en plus des jumelages (coopération décentralisée), de l'assistance technique et des remises de dettes, comprend trois formes d'interventions : les dons, les prêts sans intérêts et ceux avec intérêts (concessionnels). Parfois dirigés vers des coentreprises et plus fréquemment intégrés à un package où se croisent dons, remises de dettes et autres prêts, ces prêts concessionnels doivent être assimilés à des investissements publics. Ces derniers sont majoritairement octroyés et garantis par l'Export \& Import Bank of China (Exim, 1994), une des trois policy banks chinoises au cœur de son aide au développement. L'analyse porte ici sur ces investissements publics, les discours officiels chinois les assimilant à de la coopération. Réelles ou fantasmées, ces imbrications soulignent le nationalisme et le «capitalisme du parti-État » chinois (Bergère, 2013 ; Cabestan, 2015). Ils portent tous deux les intérêts nationaux afin d'assurer le développement endogène chinois.

Les prises de participation dans des sociétés étrangères, nommées investissements en capital social par le Trésor français (2014), sont particulièrement intéressantes. Analysés par Pairault (2010) et Richet (2013), ces investissements entrent dans la catégorie des IDE chinois. Quelles que soient les formes d'investissement (OCDE, 2008a), ces flux concernent les entreprises chinoises, que leur siège social soit situé en Chine continentale ou à Hong Kong. En dépit des différences de mesures et de définitions entre la Chine et l'OCDE, nous retenons ici deux principales sources. Il s'agit en premier lieu des flux ayant pour origine les principales entreprises publiques chinoises, sous tutelle centrale (ETC) et plus précisément celles de la State-Owned Assets Supervision and Administration Commission (SASAC, 2014), agence publique placée sous l'autorité du Conseil des affaires de l'État dirigé par le Premier ministre ; ces 112 « champions nationaux » chinois, créés par fusions-absorptions successives, sont les représentants de Pékin dans les classements mondiaux, dont le Fortune Global. En second lieu, il peut s'agir d'investissements réalisés par les 4267 entreprises chinoises à capitaux publics autorisées par le ministère du Commerce (MOFCOM, 2016) à opérer à l'étranger depuis le lancement de la Zǒuchuqu Zhànlüè ou Going out policy par Jiang Zemin en 1996 (Conseil d'État, 2006).

Les acteurs chinois présents en Afrique peuvent être classés selon leur statut, privé ou public. Les entreprises publiques sont généralement encadrées par l'Ambassade et le Bureau économique et commercial ; il s'agit de groupes à capitaux publics sous tutelles centrales (ETC) - l'État - ou locales (ETL) - les provinces - ainsi que de leurs filiales créées à l'étranger. Les employés chinois sont rémunérés par le gouvernement ou par une entreprise publique, de rang étatique 
ou provincial. Selon les recherches effectuées, seules des ETC ont, jusqu'en 2016, investi en Côte d'Ivoire.

L'ensemble de ces acteurs peut autant être impliqué dans des IDE que dans des prises de participation dans des entreprises étrangères. Nous prenons aussi en compte les coentreprises sino-ivoiriennes, qui sont une forme d'investissement public assimilée à de l'aide selon Pékin. En revanche, nous ne prenons pas en considération les prestations de service, qu'elles soient réalisées par des entreprises à capitaux publics ou privés. Cet ensemble de modalités rend compte de la dualité de la diplomatie économique chinoise, et de sa vocation commerciale. En plein essor depuis la fin des années 1990, ce vaste réseau de coopération "statocentré » recherche de nouveaux actifs, marchés et ressources, qui doivent alimenter les lignes de production de l'économie manufacturière chinoise à haute intensité de main-d'œuvre.

Bien qu'ils ne soient pas intégrés à la coopération, les flux commerciaux bilatéraux soutiennent également ce rehaussement des relations sino-ivoiriennes et sino-africaines. Prépondérants dans les échanges commerciaux de marchandises entre la Chine et la Côte d'Ivoire, les produits agricoles ivoiriens forment $83 \%$ des exportations ivoiriennes vers la Chine entre 1995 et 2014 (CNUCED, 2016). Ils sont peu variés : $99 \%$ des exportations sont constituées de seulement quatre produits : le cacao $(20 \%)$, l'hévéa $(15 \%)$, le bois $(14 \%)$ et surtout le coton $(50 \%)$. Alors que la balance commerciale est à $82,7 \%$ favorable à Pékin, ils permettent néanmoins à l'État ivoirien d'augmenter ses recettes et d'atténuer son déficit commercial avec la Chine (4,7 milliards $€$ en 2014).

Bien que la Chine ne représente que 3,7\% des échanges commerciaux globaux de la Côte d'Ivoire entre 1995 et 2014 - mais $5,7 \%$ en 2014 -, les exportations ivoiriennes ont toutefois été multipliées par 18, et les exportations chinoises, elles, par 588 ! Ces courbes commerciales suivent exactement l'évolution de l'aide chinoise, qui augmente faiblement entre 1995 et 2002, stagne entre 2002 et 2005 , et croît à partir de l'année 2005. Cette évolution des flux commerciaux n'est pas spécifique à la Côte d'Ivoire : entre la Chine et l'Afrique, exportations et importations cumulées ont été multipliées par 29 entre 1995 et 2014 (de 7,9 à 231 milliards \$), faisant de la Chine le premier partenaire commercial du continent depuis 2009. Par conséquent, commerce, aide et IDE suggèrent une diversification des acteurs chinois en Afrique, ainsi qu'une complexification des relations bilatérales et multilatérales.

Entre Pékin et Abidjan (Aurégan, 2011), les relations ont connu un véritable essor à partir de 2000 et l'arrivée au pouvoir de Laurent Gbagbo (Aurégan, 2016b). Pour l'ensemble de la période 1983-2014, on peut évaluer le nombre d'interventions chinoises en Côte d'Ivoire à 177 (Aurégan, 2016a), pour un montant total (aide et investissements) s'élevant à 4 milliards $€$. Cette somme correspond à l'aide publique au développement (APD) accordée par la France à la Côte d'Ivoire sur la période 1995-2010. Une partie de ces 4 milliards a été orientée vers le secteur agricole ivoirien.

\section{La place de l'agriculture dans la relation sino-ivoirienne}

Entre 1983 et 2014, la coopération agricole chinoise en Côte d'Ivoire représente 54 millions $€$, soit seulement 1,32\% $\mathrm{du}$ total des interventions publiques chinoises dans ce pays - tous domaines confondus. Les principaux secteurs d'intervention de la Chine restent les infrastructures $(66 \%$ du montant global), les télécommunications (18\%) ou l'éducation (6\%). De fait, la Chine populaire est en Côte d'Ivoire ce qu'elle est à l'échelle africaine : un acteur secondaire dans le secteur agricole. Cette place congrue de l'agriculture dans les interventions publiques chinoises est en contradiction avec les effets d'annonce et les discours des officiels chinois, la plaçant toujours en tête des priorités pékinoises - dans sa relation avec l'Afrique il s'entend. Depuis 2000 et le premier FOCAC, et davantage depuis 2006 et la réintroduction des centres de démonstration agricoles, la Chine érige effectivement le secteur agricole au rang 1 de ses plans d'action (Aurégan, 2015).

Diplomatiquement, médiatiquement et politiquement, l'importance accordée à l'agriculture est à corréler aux besoins locaux, puisque ce secteur est la principale source de revenus pour $68 \%$ des 22 millions d'Ivoiriens, 25 à $30 \%$ de la population active travaillant officiellement dans l'agriculture. Le secteur primaire constitue $26 \%$ du PIB ivoirien et près de $70 \%$ des recettes d'exportation. Le cacao représente en moyenne $30 \%$ des recettes annuelles d'exportation ivoiriennes entre 2007 et 2012 (BCEAO, 2014).

Compte tenu de la géographie du territoire ivoirien et de la dynamique de ses systèmes productifs (Colin, 1990), avec ses climats humides dans la partie Sud qui permettent la culture de nombreux produits d'exportation tels que le cacao (Ruf, 1997), l'anarcade, le café, la banane ou potentiellement le riz, la majorité des 19 projets agricoles chinois recensés en Côte d'Ivoire sont situés dans sa partie méridionale (Fig. 1). La coopération se répartit comme suit : dix dons, quatre prêts, quatre accords de coopération et un jumelage. Ce sont les prêts sans intérêts qui forment la majeure partie $(80 \%)$ des 29 millions $€$ alloués par Pékin entre 1983 et 2014 (Tab. 1). Les dons, nombreux mais aux montants faibles, représentent seulement $6 \%$ de cette coopération (2,3 millions $€$ ). Enfin, les accords de coopération et le jumelage entre Lakota (CentreSud) et Baoding (Hebei, Sud-Ouest de Pékin) correspondent respectivement à 1 et $12 \%$ du total de la coopération agricole bilatérale.

Si les prêts sans intérêts sont aussi prédominants, c'est principalement le fait du centre de démonstration agricole rizicole - de Guiguidou, situé à proximité de Divo dans le Sud de la Côte d'Ivoire. Il représente à lui seul $30 \%$ (11 millions $€)$ du montant de l'aide agricole chinoise. En y ajoutant les deux futurs centres de Yamoussoukro (Centre) et de Korhogo (Nord), qui coûteront 18 millions $€$ (soit $48 \%$ ), ce sont les trois quarts $(78 \%)$ de la « solidarité » chinoise qui sont orientés vers ces missions d'assistance technique agricole (29 sur 37 millions $€$ ). À terme, la Côte d'Ivoire va devenir le territoire africain le plus destinataire de cette coopération : sur les 12 centres opérationnels en Afrique subsaharienne, celui de Guiguidou est déjà le plus vaste (442 ha). L'équipe chinoise, originaire du Liaoning et employée par une entreprise publique de la même province, forme les agriculteurs locaux à la riziculture. Cinq phases ponctuent l'évolution de ces centres, de l'installation jusqu'au départ des techniciens chinois : in fine, le centre doit être autosuffisant et remis au pays d'accueil.

Par ailleurs, plusieurs interventions chinoises ont été effectuées, mais n'ont pas - encore - donné lieu à un projet matérialisé, voire ont achoppé en raison de facteurs hétérogènes 


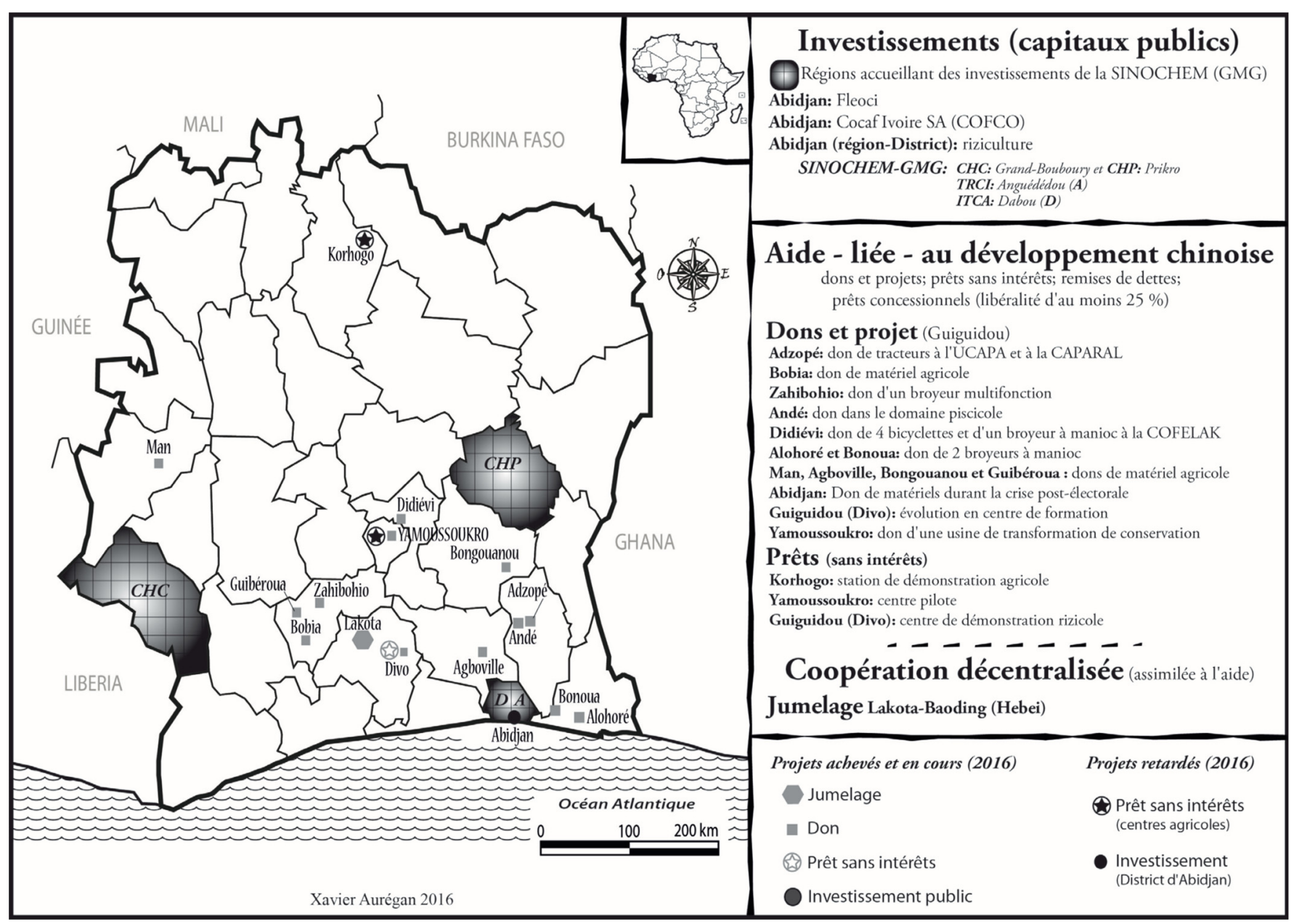

Fig. 1. Représentation spatiale des interventions agricoles chinoises en Côte d'Ivoire depuis 1983.

Fig. 1. Spatial representation of Chinese agricultural interventions in Ivory Coast since 1983.

Tableau 1. Les interventions agricoles chinoises en Côte d'Ivoire depuis 1983.

Table 1. Chinese agricultural interventions in Ivory Coast since 1983.

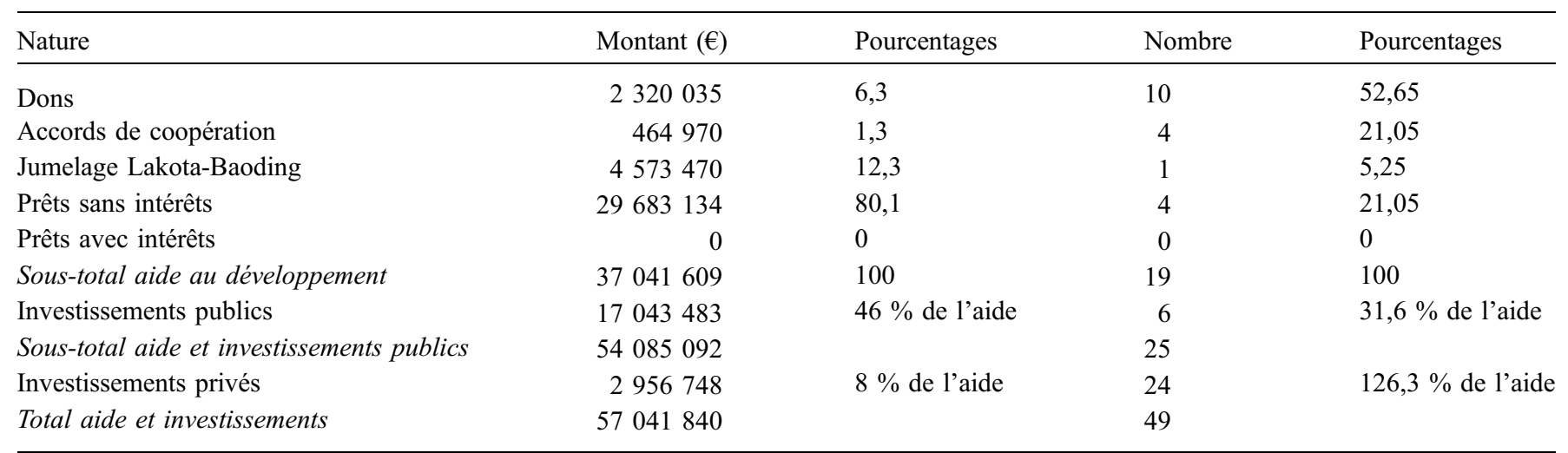

(Tab. 2). Les interventions publiques chinoises qui nous intéressent, les investissements publics sortant du cadre de l'aide, sont de l'ordre de 17 millions $€$ au total, ce qui équivaut à $46 \%$ de l'aide. À titre de comparaison, les investissements privés réalisés par des migrants-investisseurs chinois (Aurégan, 2016a) ayant créé in situ des sociétés de droit ivoirien cumulent
2,9 millions $€$ d'investissement, ce qui représente $8 \%$ du montant de l'aide.

À l'échelle internationale, $4 \%$ des IDE chinois ont pour destination le continent africain, soit l'équivalent de 2 milliards \$ par an. L'Afrique du Sud, comme pour les échanges commerciaux, en accapare un quart, suivie du Soudan (9\%) 
Tableau 2. Projets annulés ou en cours de négociation entre la Chine et la Côte d'Ivoire dans le domaine agricole.

Table 2. Cancelled projects or under negotiation between China and Ivory Coast in the agricultural sector.

\begin{tabular}{lll}
\hline Projet annulé ou retardé & État & Cause(s) \\
\hline Usine de chocolaterie en Chine (Qingke) & $\begin{array}{l}\text { Annulé (récente tentative } \\
\text { de réactivation) }\end{array}$ & Conflit entre actionnaires \\
Agro-industrie à Yamoussoukro & Annulé & L'État ivoirien ne s'est pas porté garant du partenaire privé \\
Marché de Yamoussoukro & Annulé & Financement chinois défaillant \\
Centre de Yamoussoukro & En cours de négociation & Conflits fonciers \\
Station de Korhogo & En cours de négociation & Conflits fonciers \\
Lycée agricole d'Adzopé & Annulé & Conflits politiques-fonciers \\
Barrage hydro-agricole de Moulongo & Annulé & Nécessité de service \\
Projet rizicole RIZZO de Guiglo & Annulé & L'État ivoirien ne s'est pas porté garant du partenaire privé \\
Riziculture à San Pedro & Annulé & Coût de l'aménagement et viabilité du site \\
Aménagement du district d'Abidjan & Tests en cours & Bureaucratie et manque de suivi \\
\hline
\end{tabular}

Nécessité de service : décision prise par le gouvernement ivoirien et faisant abstraction du droit. Elle peut être assimilée à un cas de force majeure.

et du Nigéria ( $8 \%$ ). Marginale, l'agriculture forme environ $2,5 \%$ du total des investissements chinois en Afrique. Néanmoins, ce secteur est de plus en plus recherché par les groupes publics chinois, puisque si ces IDE concernent majoritairement les hydrocarbures, ils se diffusent vers tous les États reconnaissant officiellement Pékin et touchent désormais tous les secteurs d'activités. Les principales entreprises d'État chinoises, ces géants enregistrés auprès de la SASAC, diversifient ainsi leur portefeuille, appuient les stratégies sectorielles étatiques et participent à leur niveau à la financiarisation de l'agriculture. De fait, plusieurs groupes chinois - COFCO en premier lieu - font partie des principaux acteurs spéculatifs. En organisant la production en fonction des marchés et en adaptant l'offre à la demande notamment, ils accélèrent la mondialisation de l'agriculture et, par conséquent, la financiarisation des matières premières et des marchés. Avec leurs logiques de compétitivité, de croissance et de rentabilité, ces groupes aux stratégies d'entreprise mondialisées entraînent l'internationalisation des marchés, fonciers ou non, et des produits agricoles. Ces groupes chinois sont certes des concurrents des firmes transnationales occidentales ADM, Bunge, Cargill et Dreyfus ( ABCD »), mais ils ne présentent en aucun cas des stratégies fondamentalement différentes.

En dépit de la pluralité des modus operandi et des types d'acteurs chinois désormais implantés en Afrique, les investissements publics chinois en Côte d'Ivoire ne concernent que deux ETC et deux filières agricoles : le cacao et l'hévéa. Le sucre, le café, la pisciculture et l'huile de palme ne font pas l'objet d'investissements par Pékin et ses acteurs économiques. Bien que non abordés ici, des conflits locaux et des procédures judiciaires sont associés à certains investissements agricoles chinois ; ils concernent ces deux filières agricoles ivoiriennes de premier plan, le cacao et l'hévéa.

\section{Les investissements dans les filières cacao et hévéa}

\subsection{Dans le cacao, une stratégie d'investissement multiscalaire avec un impact ivoirien}

Malgré de multiples réformes, d'innombrables conflits et « affaires », la filière cacao ivoirienne (Losch, 2000) reste mondialement incontournable, la Côte d'Ivoire étant considérée comme un price maker, ou faiseur de prix. Faisant suite à la Conférence mondiale du cacao, la déclaration d'Abidjan du 23 novembre 2012 a mis en avant la nécessaire durabilité de l'économie cacaoyère. Cette déclaration témoigne surtout de l'attractivité du territoire ivoirien et de ses plantations. Dans ce secteur dominé par les firmes transnationales occidentales, la Chine, d'acteur marginal, s'impose progressivement comme un investisseur capable d'exporter le cacao ivoirien. L'État chinois a investi au sein de sa région administrative spéciale, Hong Kong, plateforme « offshore » qui compte pour environ huit dixièmes de ses IDE (Pairault, 2012, 2014). Il s'agit de trois participations dans Noble Group Limited (NOBG), le plus important négociant de matières premières en Asie selon les recettes, et qui possède des intérêts dans au moins trois pays africains, dont la Côte d'Ivoire pour le cacao.

Jusqu'en décembre 2015, l'investissement chinois était matérialisé par la coentreprise NOBG-COFCO, dénommée Noble Agri, dont le capital de 1,5 milliard $\$$ était détenu à $51 \%$ par COFCO, China National Cereals, Oils and Foodstuffs Corp., puissant groupe public de Chine continentale. Dans cette joint-venture figuraient par ailleurs deux autres opérateurs « chinois », un public et un privé, formant, avec COFCO, le consortium ayant investi dans NOBG. Le public, China Investment Corporation (CIC), est un fonds souverain géré et administré par les représentants de l'État chinois. Le privé, HOPU Jinghua Investment Management Company Ltd. (Hopu Investment), est une société de capital-investissement singapourienne à capitaux propres créée en 2008 par trois Chinois, deux anciens de Goldman Sachs et un de KPMG. Ces trois acteurs chinois ont recapitalisé Noble Group en 2007 (avec la CIC) et surtout en 2014 (avec la COFCO), année de la création de Noble Agri. Fin décembre 2015, COFCO et NOBG se sont entendus pour la vente des $49 \%$ restant à l'ETC COFCO, qui est ainsi devenue détentrice de $100 \%$ de Noble Agri (Noble Agri, 2015) et a par ailleurs renommé Noble Agri en COFCO Agri en 2016.

COFCO est classée au rang 121 de Fortune Global en 2016 ; listée par la SASAC, elle a bénéficié du monopole lié aux importations et exportations alimentaires entre les années 1950 et 1980, faisant de cette entreprise stratégique le principal fournisseur de produits et services dans le secteur 
agroalimentaire chinois. Ses investissements lui ont permis d'être un acteur central dans les céréales, le vin, les produits laitiers, le thé... ou encore le chocolat. Avec l'opération de 2014, COFCO a considérablement élargi les portefeuilles de NOBG, et inversement. Leur joint-venture, Noble Agri, permet à $\mathrm{COFCO}$ de mieux contrôler les prix du marché céréalier sans passer par ABCD. La stratégie s'est vérifiée début 2014 par un autre investissement majeur (4 milliards \$, dette incluse) réalisé chez Nidera, maison de commerce néerlandaise, qui lui a donné accès au marché sud-américain. Deux jointventures ont été créées, le 23 mars 2014 pour Nidera, et le 30 septembre pour NOBG. Dans les deux cas, COFCO est devenu majoritaire et a acquis $51 \%$ du capital, avant de devenir actionnaire unique en 2016 (Nidera et COFCO Agri). Les dernières transactions, faisant passer la totalité du capital de Noble Agri et de Nidera chez COFCO, devraient vraisemblablement entraîner de nouveaux investissements, y compris dans le cacao et donc en Côte d'Ivoire. Les opérateurs chinois semblent effectivement intéressés à développer leur présence dans cette filière, en témoigne la délégation de 14 investisseurs venus s'enquérir des opportunités auprès du Conseil du cafécacao entre le 14 et le 20 avril 2016 (Conseil du café-cacao, 2016). Parmi eux, Smile Group, propriétaire de l'Afición Chocolate Village, à Jiashan, près de Shanghai, un des trois parcs d'attraction chinois mettant à l'honneur le chocolat (en sus de Pékin et Chongqing).

L'État chinois est implanté en Côte d'Ivoire via COFCO et Noble Agri, puisque en 2008, NOBG a créé une filiale locale : la société anonyme COCAF Ivoire SA. Dans cette dernière, Noble Agri - aujourd'hui COFCO Agri - détient la totalité des parts, soit 3,2 millions $€$. Bien que ces informations n'aient pu être vérifiées sur le terrain, on apprend sur son site Internet (http://www.cofcoagri.com/en/) que Noble Agri forme des producteurs de cacao en Côte d'Ivoire depuis 2010, et qu'il a depuis élargi sa zone géographique pour travailler avec « 13 groupes d'agriculteurs en Afrique de l'Ouest - qui représentent plus de 24000 petits exploitants agricoles individuels » dans la filière cacao. L'ambition de Noble Agri est de porter ce nombre à 140000 en 2020 . Noble Agri précise également qu'il entend contribuer au développement rural ivoirien par des programmes de formation et de certification, et affirme travailler avec la World Cocoa Foundation (WCF) financée par la Fondation Bill et Melinda Gates et douze groupes industriels, afin d'appuyer 200000 ménages en Afrique de l'Ouest (Libéria, Côte d'Ivoire, Ghana et Nigéria) et au Cameroun (Noble Group, 2009). Enfin, souhaitant mettre en avant son implication en termes de responsabilité sociale des entreprises (RSE), Noble Group a financé la bourse Noble Cocoa, soutenant 30 mères de famille par des microcrédits pour leur permettre de fonder des petites entreprises à Gribouo, à l'Ouest de la Côte d'Ivoire, entre Soubré et Guiglo (Noble Group, 2015).

COCAF Ivoire SA, dont la part de marché représente entre 5 et $7 \%$ du marché local selon les années (environ 74000 t de cacao sur un total de 1,1 million) et dont le siège social se trouve à Abidjan, fait partie des 53 sociétés commerciales (sur 95 au total, sociétés coopératives comprises) agréées en qualité d'exportateurs de café-cacao pour la campagne 2014-2015 (Conseil du café-cacao, 2014). Par ailleurs, COCAF est, avec d'autres exportateurs secondaires, membre $\mathrm{du}$ Groupe des négociants internationaux (GNI). Jusqu'en
2014, ce lobby était piloté par l'ancien groupe Armajaro Trading Limited, dont le directeur pour l'Afrique était Loïc Folloroux, beau-fils du président ivoirien Alassane Ouattara, et le cofondateur Anthony Ward, surnommé «Chocolate Finger » pour ses spectaculaires opérations spéculatives : 300000 t en 1996 (10\% de la production annuelle mondiale), $148000 \mathrm{t}$ de cacao ivoirien en 2002 après la tentative de coup d'État à Abidjan, et 240000 t en 2010 (15\% des stocks). Il a cédé ses activités de trading (cacao, sucre et café) en 2013. Toujours en activité à Abidjan, le GNI, quant à lui, tente de faire pression sur les autorités ivoiriennes afin d'affaiblir les principaux leaders du marché ivoirien, tant Cargill que Nestlé. En définitive, la création d'une filiale locale, en l'espèce COCAF, apporte deux enseignements. D'une part, il semblerait que les investisseurs chinois aient apprivoisé le cadre concurrentiel et pour partie oligopolistique de cette filière en contournant les principaux acteurs déjà en place. D'autre part, en s'associant avec d'autres dans le GNI, COFCO semble maîtriser les rivalités de pouvoir locales et sectorielles, en participant à la création d'un contre-pouvoir censé rebattre les cartes d'un produit d'exportation phare de la sous-région.

Si le cacao ivoirien (Losch, 2000 ; Global Witness, 2007) est convoité par un géant de l'agroalimentaire chinois et mondial, que dire de l'hévéa et du représentant chinois, en situation de quasi-monopole jusqu'aux années 2000 ?

\subsection{La stratégie hévéicole ivoirienne d'un pétrolier chinois}

Le secteur de l'hévéa représente une niche économique d'avenir pour la Chine. C'est, avec le riz (coopération bilatérale), les ressources halieutiques et le bois (investissements privés), le principal produit agricole où la présence d'acteurs chinois est manifeste.

Avec 2,5\% de l'hévéa exporté par la Côte d'Ivoire entre 1995 et 2014 (CNUCED, 2016), soit 199 millions sur 7,7 milliards \$, la Chine et Singapour cumulés ne semblent pas figurer parmi les principaux partenaires des ports autonomes d'Abidjan et de San Pedro. Pourtant, la filiale locale, GMG-CI, du groupe GMG, lui-même filiale de SINOCHEM, contrôle majoritairement puis partiellement trois opérateurs que sont l'Ivoirienne de traitement du caoutchouc (ITCA), Tropical Rubber Côte d'Ivoire (TRCI), et les entités Compagnie hévéicole du Cavally-Compagnie hévéicole de Prikro (CHC-CHP) de la Société d'investissement pour l'agriculture tropicale (SIAT). Par ailleurs, GMG est associé à deux conflits sociopolitiques ivoiriens qui nuisent largement à l'ensemble des acteurs de la filière.

Entreprise d'État sous tutelle centrale listée par la SASAC, SINOCHEM est un des plus puissants groupes chinois. Avec plus de 300 filiales en Chine et à l'étranger, SINOCHEM a été fondé en 1950 au lendemain de la proclamation de la République populaire de Chine. Plus important groupe de négoce chinois, il fait partie des quatre entreprises pétrolières d'Etat, ce qui ne l'empêche pas d'ouvrir son capital, désormais contrôlé à $2 \%$ par un autre géant public chinois spécialisé dans le transport de marchandises, COSCO.

Constituée en 1999 à Singapour, GMG Global Ltd. est une société spécialisée dans le caoutchouc naturel. Elle est particulièrement active en Afrique francophone (Côte d'Ivoire, 
Cameroun et République démocratique du Congo) et en Asie du Sud-Est (Thailande et Indonésie) - où se situe la production mondiale d'hévéa. Au total, elle revendique environ 78000 ha de plantations pour une production annuelle de 527000 t. En juillet 2008, SINOCHEM acquiert $51 \%$ des actions de GMG. Le 4 novembre 2008, GMG est admise au Singapore Exchange (SGX). Après plusieurs investissements en Indonésie et en Thaïlande induits par la recapitalisation, GMG investit en Côte d'Ivoire à travers deux partenaires : ITCA et SIAT. GMG dispose d'une filiale à Abidjan, la société anonyme avec conseil d'administration GMG-Côte d'Ivoire (GMG-CI), au capital social de 1,5 million $€$ et domiciliée à Abidjan, dans les mêmes locaux que l'ITCA.

En juillet 2011, GMG acquiert donc $60 \%$ des parts de l'ITCA pour environ $450000 €$. L'augmentation du capital social est actée le 24 février 2012, quelques jours après la cession d'actions au profit de cinq personnes physiques, dont Tien Sing Young, PDG de GMG-CI, et une personne morale, GMG Global. En février 2012, la société anonyme ITCA devient une SA avec conseil d'administration ; Yao Xingliang, ex de SINOCHEM, est nommé président du conseil d'administration, et l'ancien ministre et conseiller présidentiel Vincent Essoh Lohoues, directeur général.

Le 21 février 2012, GMG Global Ltd. acquiert $35 \%$ de la SIAT, présidée et financée par l'industriel belge Pierre Vandebeeck - à travers la holding familiale Fimave. Avant la cession, ce dernier détenait alors $83 \%$ des parts pour 193 millions $€$. Créée en 1991 lorsqu'elle acquiert 3000 ha de palmiers à huile au Nigéria, la SIAT a ensuite investi au Ghana, au Gabon et enfin en Côte d'Ivoire. Elle revendique 55000 ha de plantations de palmiers à huile et 16000 d'hévéas, en sus de trois complexes industriels d'huile de palme (Nigéria, Ghana et Gabon). L'investissement chinois, via la GMG, permet au groupe belge d'accéder au territoire camerounais, où GMG Global détient $90 \%$ de l'HEVECAM (Société d'hévéa du Cameroun, 41000 ha et une usine de transformation), et de nouveau à la Côte d'Ivoire, où GMG à également investi dans TRCI $(51,2 \%$ du capital, 1560 ha et une usine de transformation de $36000 \mathrm{t}$ annuelles). De fait, la SIAT accède désormais et par cette cession au marché chinois et se rapproche de l'un de ses principaux clients (Michelin, également présent en Côte d'Ivoire).

Par ses investissements ciblés en Côte d'Ivoire, SINOCHEM contrôle en définitive $20 \%$ du marché hévéicole ivoirien. Il pourrait rapidement devenir un acteur incontournable de cette « innovation hévéa » (Ruf, 2012) qui, du fait du vieillissement des plantations caféières, doit ravir la place du café dans l'historique binôme café-cacao ivoirien.

Les investissements des ETC chinoises via GMG et via Noble Group n'étant jamais mentionnés dans les articles de la presse ivoirienne ou régionale, leurs réputations (Tribunal de commerce d'Abidjan, 2014) ainsi que celle de la Chine ne sont jamais entachées quand des scandales financiers touchent ses filières. Que ces investissements soient ou non décriés, deux éléments méritent d'être signalés. Premièrement, ces filières sont accaparées par les acteurs ouest-africains et occidentaux ; les opportunités d'investissement sont donc somme toute limitées. Secondement, il semble peu plausible que les conflits et les procédures judiciaires ivoiriens des filières cacao et hévéa inquiètent des groupes et des acteurs habitués aux rapports de force et de puissance chinois, tout aussi violents.

\section{Conclusion}

En Côte d'Ivoire, l'agriculture devient progressivement un des secteurs intéressant la Chine et ses acteurs, publics comme privés. Cela traduit autant les attentes locales, africaines, que les volontés chinoises en la matière. Depuis la création de la SASAC en 2003, les puissants groupes publics investissent de plus en plus sans avoir directement recours à l'aide au développement chinoise, en développant des stratégies commerciales et financières identiques aux firmes occidentales. À un détail près tout de même, puisque ces ETC s'insèrent dans un enchevêtrement complexe où influences économiques et politiques gouvernementales forment une toile dans laquelle demeure une constante, le président de la SASAC est toujours un membre du Comité central du Parti communiste chinois.

Dans une région ouest-africaine historiquement liée à la France et à ses opérateurs, ces " premiers " investissements agricoles et ces deux acteurs publics chinois (COFCO et SINOCHEM) illustrent l'internationalisation des entreprises à capitaux publics chinoises, et leurs objectifs commerciaux. Entre ouverture, protectionnisme et stratégies étatiques, ces actionnaires majoritaires de plusieurs groupes asiatiques et ivoiriens développent, délibérément ou non, une politique de sécurisation et d'accès aux produits alimentaires. Des ordres, des conseils avisés ou des directives sont-ils donnés par le Conseil des affaires de l'État qui coiffe la SASAC et/ou les ministères ? Toujours est-il qu'en investissant, ils mettent en œuvre une partie des prérogatives de l'État chinois, à savoir exercer une influence croissante sur les marchés agricoles mondiaux et les prix, à l'image de l'ensemble des matières premières considérées comme stratégiques par Pékin.

Les rhétoriques diplomatiques et politiques promouvant le secteur agricole lors des FOCAC sont suivies de faits concrets. L'agriculture et la sécurité alimentaire figurent en tête de la "coopération économique " sino-africaine telle que mentionnée lors des derniers FOCAC. Au point 3.1.8 du plan d'action de 2015, Pékin précise d'ailleurs que « la partie chinoise encouragera et soutiendra l'investissement des entreprises chinoises dans le domaine agricole en Afrique » (FOCAC, 2015). En cela, les IDE de SINOCHEM et de COFCO peuvent être perçus comme des engagements et placements stratégiques, soit des resource-seeking investments ou investissements à la recherche de ressources (Gelb, 2005). Dans ces cas de figure, les conflits locaux africains (Bouquet, 2008) peuvent être vus comme des coïncidences malheureuses, pour lesquelles la presse ne mentionne jamais la Chine, mais plutôt les filiales hongkongaise ou singapourienne qui servent, en quelque sorte, d'utiles paravents. Qu'en sera-t-il lorsque la Chine et/ou ses ETC seront nommément citées à la faveur d'investissements croissants dans cet État ouest-africain qui enregistre les plus hauts taux de croissance économique en Afrique depuis 2012 ?

Une difficulté réside dans l'analyse des finalités de ces stratégies commerciales chinoises: sont-elles uniquement commerciales et liées à la diversification afférente, ou sontelles pilotées depuis les ministères et le Conseil d'État ? Pour nous, compte tenu de la faiblesse relative du ministère des Affaires étrangères vis-à-vis du ministère du Commerce, de la relative fragmentation de l'État et des rivalités internes, les ETC qui ont investi en Côte d'Ivoire sont en premier lieu 
motivées par « une maximalisation de leur profit » (Cabestan, 2015). Subsidiairement, elles sont poussées à développer des lignes d'approvisionnement transcontinentales pour alimenter la dorénavant mondialisée « China Corp. ».

\section{Références}

Aurégan X. 2011. La Chine en Côte d'Ivoire : le double jeu [en ligne]. Diploweb. Disponible sur http://www.diploweb.com/La-Chine-enCote-d-Ivoire-le.html.

Aurégan X. 2013. La Chine, d'un État honni à incontournable en Côte d'Ivoire. Croisements 3: 86-111.

Aurégan X. 2015. Les enjeux du Forum de coopération Chine-Afrique [en ligne]. Diploweb. Disponible sur http://www.diploweb.com/ FOCAC-Les-enjeux-du-Forum-de.html.

Aurégan X. 2016a. Aide, investissements et migrants-investisseurs : les interventions chinoises en Côte d'Ivoire. Autrepart 76: 89-108.

Aurégan X. 2016b. Géopolitique de la Chine en Côte d'Ivoire. Paris : Riveneuve éditions.

BCEAO. 2014. Étude monographique sur la filière cacao dans l'UEMOA [en ligne]. BCEAO, p. 33. Disponible sur http://www. bceao.int/IMG/pdf/etude_monographique_sur_la_filiere_cacao_ dans_1_uemoa.pdf.

Bergère M-C. 2013. Chine : le nouveau capitalisme d'État. SaintAmand-Montrond : Fayard.

Bouquet C. 2008. Géopolitique de la Côte d'Ivoire - Le désespoir de Kourouma. Vottem : Armand Colin.

Brautigam D. 2015. Will Africa need China? Oxford: Oxford University Press.

Cabestan J-P. 2015. La politique internationale de la Chine. Entre intégration et volonté de puissance. Paris : Presses de Sciences Po.

Chaponnière J-R. 2011. Les investissements agricoles de la Chine : une source d'inquiétudes ? Afrique contemporaine 237: 71-83. doi: $10.3917 /$ afco.237.0071.

Chaponnière J-R, Gabas J-J, eds. 2012. Le temps de la Chine en Afrique - Enjeux et réalités au Sud du Sahara. Paris : Karthala.

CNUCED. 2016. Centre de données [en ligne]. In : Conférence des Nations unies sur le commerce et le développement, Genève. Disponible sur http://unctadstat.unctad.org/wds/ReportFolders/ reportFolders.aspx?sCS_ChosenLang=fr.

Colin J-P. 1990. La dynamique des systèmes productifs agricoles en basse Côte d'Ivoire. In : Dynamique des systèmes agraires. La dimension économique. Paris : ORSTOM-CNRS, p. 165-190.

Conseil du café-cacao. 2014. Décision $\mathrm{n}^{\mathrm{o}} 012 / 14$ portant agrément d'exportateurs de café-cacao au titre de la saison 2014/2015 [en ligne]. Disponible sur http://www.conseilcafecacao.ci/docs/Decision_portan t_agrement_d\%27exportateurs_de_Cafe_Cacao.pdf.

Conseil du café-cacao. 2016. Le conseil du café-cacao reçoit une délégation d'hommes d'affaires chinois [en ligne]. Disponible sur $\mathrm{http}: / /$ www.conseilcafecacao.ci/index.php?option=com_k2\&view= item\&id=589:le-conseil-du-cafe-cacao-recoit-une-delegation-d $\%$ E2\%80\%99hommes-d\%E2\%80\%99affaires-chinois\&Itemid $=18$.

Conseil d'État de la République populaire de Chine. 2006. 更好地方施 '走出去”战略 [en ligne, en chinois]. Disponible sur http://www.gov.cn/node_11140/2006-03/15/content_227686. htm.

Exim Bank of China. 1994. Preferential loan [en ligne]. Available from http://english.eximbank.gov.cn/tm/en-TCN/index_640.html.

FOCAC. 2015. Forum sur la coopération sino-africaine plan d'action de Johannesburg (2016-2018) [en ligne]. Pékin, RPC. Disponible sur http://www.focac.org/eng/ltda/dwjbzjjhys_1/t1327961.htm.
Gabas J-J. 2014. La Chine est-elle un accapareur de terres en Afrique ? Retour sur une réalité mal acceptée. Futuribles 398: 25-36.

Gelb S. 2005. South-South investment: the case of Africa [en ligne]. Africa in the world economy - The National, Regional and International Challenges Fondad, La Hague, p. 200-205. Available from www.fondad.org.

Global Witness. 2007. Chocolat chaud : comment le cacao a alimenté le conflit en Côte d'Ivoire [en ligne]. Washington : Global Witness. Disponible sur https:/www.globalwitness.org/documents/12753/ cotedivfrench.pdf.

Hellendorff B. 2012. Ressources naturelles, conflits et construction de la paix en Afrique de l'Ouest [en ligne]. Bruxelles: GRIP. Disponible sur http://www.grip.org/sites/grip.org/files/RAP PORTS/2012/Rapport_2012-7.pdf.

Losch B. 2000. Coup de cacao en Côte d'Ivoire - Économie politique d'une crise structurelle. Critique internationale 1: 6-14.

MOFCOM. 2016. List of China's qualified overseas contractors [en ligne, page visitée le 23 septembre 2016]. Pékin, RPC. Available from http://wszw.hzs.mofcom.gov.cn/fecp/zsma/corp/corp_ml_lis t_en.jsp?selmanage=\&corp_name=http://wszw.hzs.mofcom.gov. cn/fecp/zsma/corp/corp_ml_list_en.jsp?selmanage=\&corp_name.

Noble Agri. 2015. COFCO International Ltd. Acquires full ownership of Noble Agri Ltd [en ligne]. Available from http://www.cofcoagri. com/en/cofco-agri-news/news/cofco-international-ltd-acquiresfull-ownership-of-noble-agri-ltd.

Noble Group. 2009. 60,000 farmers to benefit from cocoa livelihoods programme [en ligne]. Available from http://thisisnoble.com/in-thepress/249-60000-farmers-to-benefit-from-cocoa-livelihoods-pro gramme.html.

Noble Group. 2015. Annual report 2015 [en ligne]. Available from $\mathrm{http}$ //thisisnoble.com/images/investors/financialInformation/ annualReport/ar2015.pdf.

OCDE. 2008a. Définition de référence de l'OCDE des investissements directs internationaux [en ligne]. $4^{\mathrm{e}}$ éd. Paris : Organisation de coopération et de développement économiques, 288 p. Disponible sur http://www.oecd.org/fr/industrie/inv/statistiquesetanalysesdelin vestissement/40632182.pdf.

OCDE. 2008b. Recommandation du cas sur le déliement de l'aide [en ligne]. Paris : OCDE, 16 p. Disponible sur http://www.oecd.org/fr/ cad/41708406.pdf.

Pairault T. 2010. Le rôle des investissements directs étrangers entrants et sortants en Chine : une appréciation [en ligne]. Région et Développement 311: 119-142. Disponible sur https://halshs. archives-ouvertes.fr/halshs-00982768/document.

Pairault T. 2012. Sur l'investissement chinois en Afrique [en ligne]. Chine-Afrique. Disponible sur http://www.pairault.fr/sinaf/index. php/l-investissement-chinois-en-afrique.

Pairault T. 2014. Chinese direct investment in Africa. A state strategy? [en ligne]. Région et Développement 37: 259-284. Disponible sur https://halshs.archives-ouvertes.fr/halshs-00981417/document.

Richet X. 2013. Internationalisation des firmes chinoises : croissance, motivations, stratégies [en ligne]. In : Séminaire BRICs, UP3 université Sorbonne nouvelle, 21 p. Disponible sur https://halshs. archives-ouvertes.fr/halshs-00796197.

Ruf F. 1997. Booms et crises du cacao. Les vertiges de l'or brun. Paris : Karthala.

Ruf F. 2012. L'adoption de l'hévéa en Côte d'Ivoire. Prix, mimétisme, changement écologique et social. Économie rurale 330-331: 103-124.

SASAC. 2014. Liste des entreprises centrales [en ligne]. Pékin, RPC : SASAC. Disponible sur http:/www.sasac.gov.cn/n1180/n1226/ n2425/. 
Trésor français. 2014. Les investissements directs à l'étranger (IDE) - définition [en ligne]. Paris : Direction générale du Trésor. Disponible sur http://www.tresor.economie.gouv.fr/8235_les-inves tissements-directs-a-l-etranger-ide.
Tribunal de commerce d'Abidjan. 2014. Audience publique du 31 juillet 2014 [en ligne]. Disponible sur http://www.tribunalcom merceabidjan.org/documents/publications/societe/RG17282014. pdf.

Citation de l'article : Aurégan X. 2017. Les investissements publics chinois dans les filières agricoles ivoiriennes. Cah. Agric. $26: 15003$. 